\title{
Prenatal diagnosis of junctional epidermolysis bullosa associated with pyloric atresia
}

\author{
V Nazzaro, U Nicolini, L De Luca, E Berti, R Caputo
}

\begin{abstract}
Prenatal diagnosis of junctional epidermolysis bullosa associated with pyloric atresia was carried out in a couple at risk. Their two previous children had died during the first months of life of the same disorder despite surgery for the pyloric abnormality. Ultrastructural study of fetal skin biopsies obtained at 18 weeks' gestation showed dermal-epidermal separation at the lamina lucida level, while ultrasound showed marked stomach dilatation. Light microscopy of pyloric tissue obtained after termination showed the pyloric lumen to be replaced by loose connective tissue with no inflammatory reaction. Immunofluorescence studies on the skin specimens with the monoclonal antibody GB3, known to be absent in 'lethal' junctional epidermolysis bullosa skin, disclosed a marked positivity suggesting that the junctional epidermolysis bullosa in this case may be of the 'non-lethal' type.
\end{abstract}

The association of epidermolysis bullosa and pyloric atresia is a rare but distinct entity inherited as an autosomal recessive trait. Five sib pairs ${ }^{12}$ and about 15 sporadic cases $^{1-3}$ have been reported, most of whom have died in the first few months of life despite surgical management of the pyloric abnormality. Ultrastructural studies of the skin, performed in the majority of recently reported cases, showed findings consistent with junctional epidermolysis bullosa. ${ }^{1-3}$

I Clinica Dermatologica, University of Milan, Via Pace 9, 20122 Milano, Italy.

V Nazzaro, E Berti, R Caputo

Clinica Ostetrica e Ginecologica, University of Milan, Italy.

U Nicolini

Ospedale di Tropea, Italy.

L De Luca

Correspondence to Dr Nazzaro.

This paper was presented at the International Epidermolysis Bullosa Symposium, London, 19 and 20 January 1989.

Received for publication 14 July 1989.

Revised version accepted for publication 21 November 1989.
We now report the first prenatal diagnosis of epidermolysis bullosa associated with pyloric atresia performed by ultrastructural examination of fetal skin biopsies in a couple at risk, whose previous son and daughter had died of the same combined disorder.

\section{Case report}

A 25 year old woman, gravida 4, para 3, was referred for prenatal diagnosis of epidermolysis bullosa (EB) associated with pyloric atresia (PA).

Her first pregnancy ended with the delivery at 34 weeks' gestation of a $2150 \mathrm{~g}$ girl affected with EB and PA. No polyhydramnios was recorded. Pyloroplasty was performed but the infant died at 4 days of age. No necropsy or skin biopsy were performed.

In the next pregnancy she delivered an unaffected newborn. She later had a pregnancy complicated by polyhydramnios which ended with the delivery at 38 weeks' gestation of a $3000 \mathrm{~g}$ boy affected with EB and PA. Gastroduodenostomy was performed and light microscopy of sections of the pylorus showed that the lumen was obliterated by loose connective tissue. Skin electron microscopy showed a cleavage at the lamina lucida level, between basal keratinocytes and lamina densa. The infant died of sepsis at 3 months of age and there was no necropsy.

No other family members had a history of congenital abnormalities or skin disease and the parents were not related.

Prenatal diagnosis was requested by the parents in a subsequent pregnancy.

\section{Methods}

Fetal skin biopsies were obtained at 18 weeks' gestation by transabdominal, ultrasound guided fetal skin biopsy. 21G biopsy forceps were used, introduced into the amniotic cavity through a $18 \mathrm{G}$ needle. Five specimens were taken from the fetal trunk and scalp.

For light and electron microscopy the biopsies were fixed for two hours at $22^{\circ} \mathrm{C}$ in half strength Karnovsky fixative in cacodylate buffer, post-fixed in $2 \%$ osmium tetroxide, dehydrated in ethanol, and embedded in Epon. One $\mu \mathrm{m}$ semithin sections were cut and stained 


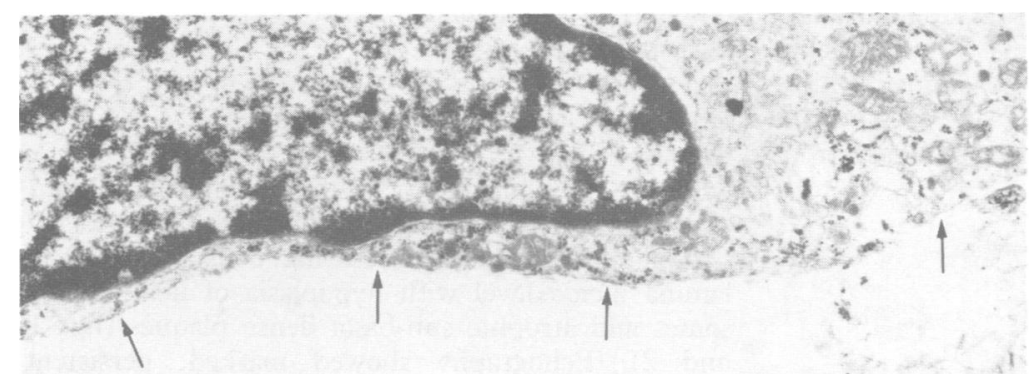

Figure 1 Electron micrograph of fetal skin biopsy obtained at 18 weeks' gestation showing separation between the basal cell plasma membrane (arrows) and lamina densa (open arrows). The lamina densa covers the dermal surface of the split.

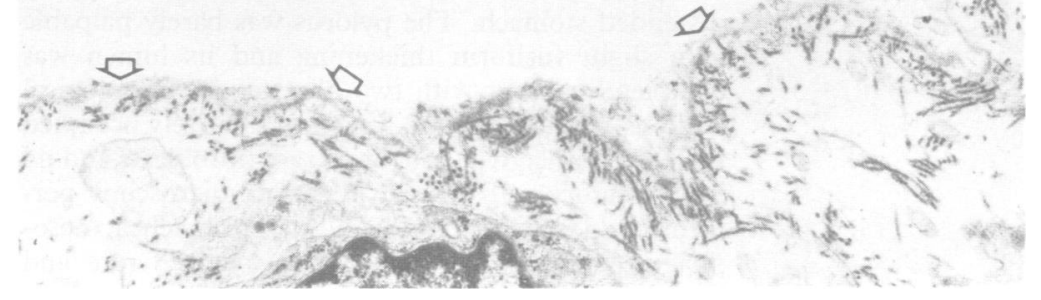

with toluidine blue. Ultrathin sections were stained with aqueous uranyl acetate and lead citrate before being examined with a Philips CM 10 transmission electron microscope.

For immunofluorescence the specimens were orientated in OCT compound and snap frozen in

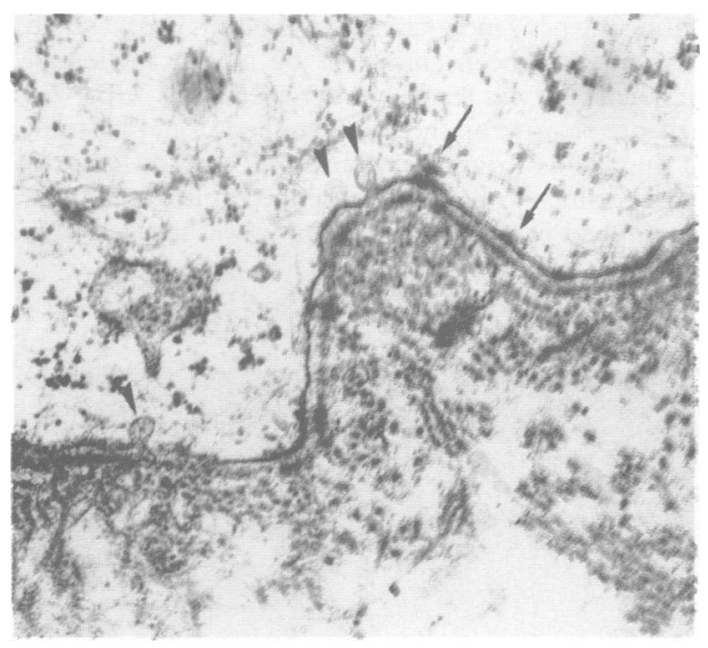

Figure 2 Electron micrograph of fetal skin biopsy obtained at 18 weeks' gestation showing unseparated skin with rare and hypoplastic hemidesmosomes and atrophic sub-basal dense plaques (arrows). Invaginations of the plasma membranes within the cytoplasm of basal keratinocytes were frequently observed (arrowheads). liquid nitrogen. Five $\mu \mathrm{m}$ thick cryostat sections, collected on glass slides and air dried, were incubated with GB3 monoclonal antibody (Sera-Lab, Sussex, England) diluted $1: 10$ in phosphate buffered saline (PBS) for 60 minutes. Parallel sections were incubated with bullous pemphigoid serum diluted 1:4 in PBS, with a rabbit anti-laminin serum (Sanbio Uden, The Netherlands) diluted 1:50 in PBS, and with anti-type IV collagen monoclonal antibody (ICN Biomedicals, Bucks, England) diluted 1:10 in PBS. After washing, the sections were incubated with fluorescein con-

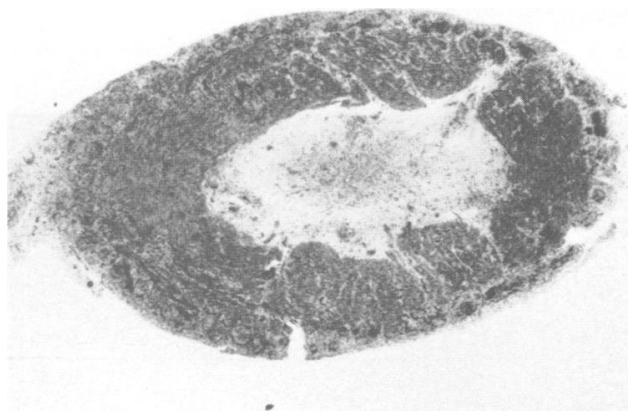

Figure 3 Light micrograph of semithin sections obtained from the pylorus of the aborted fetus. The pyloric lumen is totally obliterated by loose connective tissue and no luminal mucosa can be identified. 
jugated $F\left(a b^{\prime}\right) 2$ fragments of rabbit IgG to mouse IgG $(\mathrm{H}+\mathrm{L})$ or swine IgG to rabbit $\mathrm{IgG}(\mathrm{H}+\mathrm{L})$ (Dako, Glostrup, Denmark) before being examined with a fluorescence microscope.

Skin and gastrointestinal sections obtained from

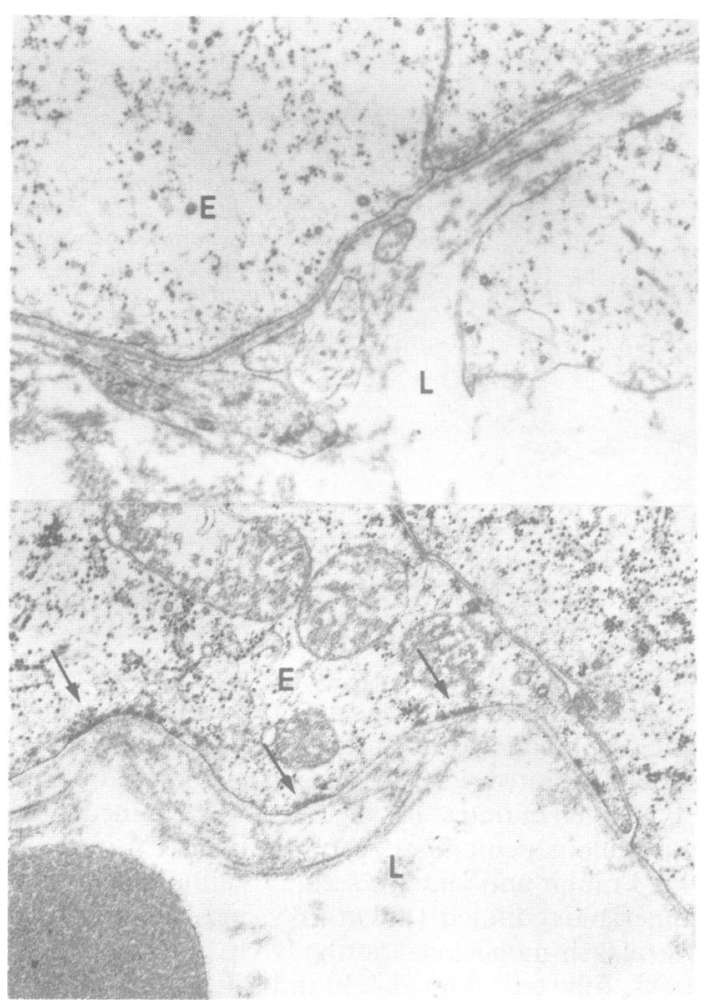

Figure 4 Electron micrographs of stomach specimens obtained from the affected aborted fetus (top) and from a normal age matched fetus (bottom). In the specimen from the aborted fetus, the hemidesmosome-like structures are rare and hypoplastic in comparison with normal hemidesmosomes as seen in the control (arrows). (E=epithelial cell, L=lamina propria). two normal, age matched fetuses were also examined as negative controls.

\section{Results}

Semithin sections from all the specimens showed dermal-epidermal separation. Ultrastructural examination showed a split occurring constantly at the lamina lucida level with hypoplasia of hemidesmosomes and atrophic sub-basal dense plaques (figs 1 and 2). Echography showed marked, persistent stomach dilatation.

After diagnosis, elective termination of pregnancy was performed. Gross examination of the fetus showed superficial skin erosions and a markedly distended stomach. The pylorus was barely palpable as a slight fusiform thickening and its lumen was completely absent with two blind ends. Histological sections showed that the lumen was entirely occupied by loose connective tissue with rare fibroblasts and no inflammatory cells (fig 3). Electron microscopy performed on specimens obtained from trachea, oesophagus, stomach, and duodenum showed rare and atrophic hemidesmosome-like structures but no junctional mucosal separation (fig 4). Ultrastructural controls of skin samples taken from the back, limbs, and scalp confirmed the findings observed prenatally.

Immunofluorescence mapping studies were performed in skin specimens with antibodies against bullous pemphigoid, laminin, and type IV collagen. Bullous pemphigoid was found at the roof of the cleavage (fig 5, left), while laminin and type IV collagen were found at the base (fig 5, centre), consistent with a split within the lamina lucida. In addition, a new monoclonal antibody (GB3), which has previously been shown to be absent in 'lethal' junctional EB, was tested. In our specimens, GB3 was markedly positive on the skin obtained from the trunk and thigh (fig 5, right) without significant differences from specimens obtained from the same areas from age matched controls. GB3 staining was also positive

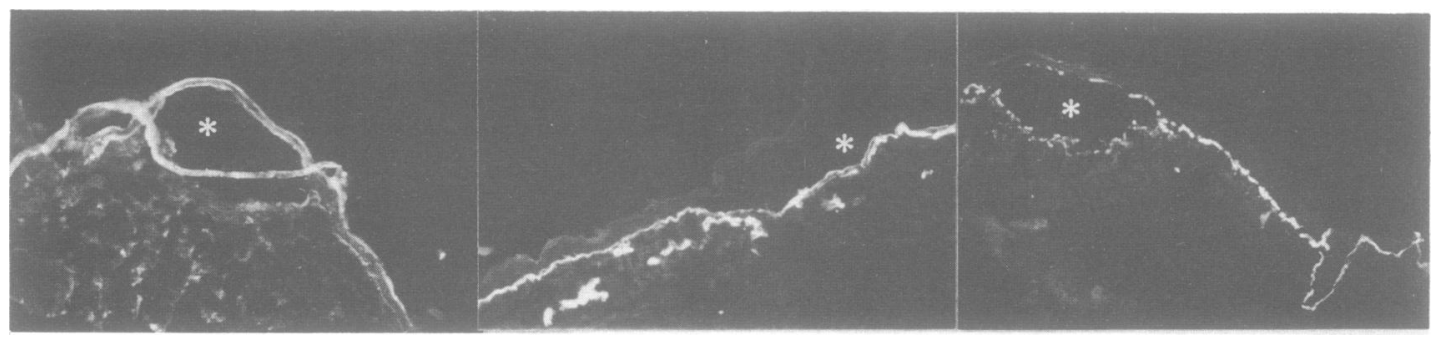

Figure 5 Immunofluorescence micrographs of skin specimens obtained from the aborted fetus. Bullous pemphigoid serum stained the roof of the blister (left), monoclonal antibody anti-type IV collagen stained the base of the blister (centre), and the monoclonal antibody $G B 3$ showed intense labelling of the epidermal basal membrane, both at the bottom and top of the blister cavity (right). ${ }^{*}=$ blister cavity. 


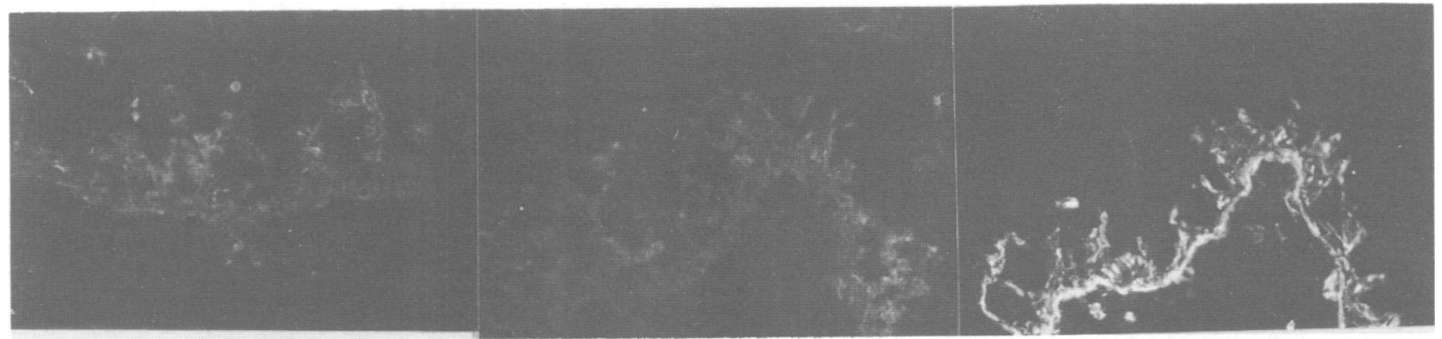

Figure 6 Immunofluorescence micrographs of stomach specimens obtained from the aborted fetus and from a normal age matched fetus. No labelling of the specimens from the aborted fetus (left) or from the normal fetus (centre) was seen with the monoclonal antibody GB3. Note, in comparison, normal staining of the same specimen from the normal fetus with anti-type IV collagen monoclonal antibody (right).

on gastrointestinal mucosa, except the stomach and pylorus (fig 6).

\section{Discussion}

Epidermolysis bullosa represents a heterogeneous family of hereditary mechanobullous diseases in which minimal trauma results in blister formation. EB is classified into three major groups according to the ultrastructural level of cleavage: simplex (epidermolytic), junctional, and dystrophic (dermolytic). Junctional EB is characterised by a cleavage through the lamina lucida of the epidermal basal membrane and is composed of at least two distinct clinical subtypes, one in which patients die in the first months of life, known as 'lethal' junctional EB or Herlitz's disease, and another in which patients are able to survive into adulthood, known as 'non-lethal' junctional EB or generalised atrophic benign EB. ${ }^{4}$ Both types present the same ultrastructural findings, are inherited as an autosomal recessive trait, and are clinically characterised by blisters that heal with atrophy but with no milia or scarring.

In our case and in the majority of recently reported cases, the EB associated with pyloric atresia has been ultrastructurally shown to be of the junctional type. ${ }^{1-3}$ The electron microscopic study performed both in one of the previous affected patients and in the fetal skin specimens disclosed a cleavage in the plane of the lamina lucida with hypoplasia of hemidesmosomes and atrophic sub-basal dense plates.

Pyloric atresia is a rare congenital anomaly representing about $1 \%$ of all gastrointestinal tract atresia. It is transmitted as an autosomal recessive trait and can result from a failure of the tube to canalise during development or a mechanical or vascular injury to the previously normal fetal intestine. ${ }^{5}$

Some authors have proposed that the pyloric obstruction in this syndrome results from intrauterine mucosal damage within junctional EB with subsequent peptic digestion and inflammatory scarring reaction. ${ }^{6}$ Our findings do not agree with this hypothesis. Histological study of the obliterated pyloric lumen at 18 weeks' gestation showed only mucoid or loose connective tissue with no inflammatory reaction.

Another, more likely, hypothesis is that this association results from the action of two closely linked genes or from the pleiotropic expression of a single abnormal gene. This is supported by the fact that no segregation of the two traits was observed in our family nor in the other six families reported so far. ${ }^{12}$ Autosomal recessive inheritance is suggested by the occurrence of this disorder in opposite sex sibs in our case and in previous reports ${ }^{12}$ and in children born to consanguineous parents in previous reports. ${ }^{2}$

The fatal outcome frequently observed in this syndrome could result from the EB alone or from the combination of EB and pyloric atresia. Pyloric atresia without EB carries a good long term prognosis with early operative management. In contrast, most of the infants affected with pyloric atresia and EB died despite early surgical intervention on the pylorus. In these cases the EB was nearly always 'lethal' or Herlitz type even though the fatal outcome could have resulted from a 'non-lethal' EB complicating the postoperative management of the gastrointestinal abnormality.

Ultrastructural study of fetal skin biopsies obtained at 18 weeks' gestation by fetoscopy or under direct echographic control is now a well established method for prenatal diagnosis of EB. ${ }^{j}$ As shown by the present study, in the association of EB with pyloric atresia careful ultrasound study of the stomach may suggest the diagnosis, which must be confirmed by ultrastructural study of fetal skin biopsy. Recently a new monoclonal antibody (GB3) that recognises an antigen(s) within the lamina lucida has been reported not to be expressed or poorly expressed in 'lethal' junctional EB skin and has been used in confirming the prenatal diagnosis of this disease. ${ }^{89}$ In our case we observed a strong positivity of GB3 at the dermal- 
epidermal junction at 18 weeks of gestation. This may indicate that the junctional EB was of the 'non-lethal' type. However, the interpretation of this finding awaits further studies on the antigenic composition of the epidermal basal membrane zone. At present, electron microscopy seems to be the only reliable method of prenatal diagnosis of this disease.

1 Egan N, Ward R, Olmstead M, Marks JG. Junctional epidermolysis bullosa and pyloric atresia in two siblings. Arch Dermatol 1985;121:1186-8.

2 Rosenbloom MS, Ratner M. Congenital pyloric atresia and epidermolysis bullosa lethalis in premature siblings. $\mathcal{F}$ Pediatr Surg 1987;22:374-6.
3 Berger TG, Detlefs RL, Donatucci CF. Junctional epidermolysis bullosa, pyloric atresia and genitourinary disease. Pediatr Dermatol 1986;3:130-4.

4 Hintner $H$, Wolff $K$. Generalized atrophic benign epidermolysis bullosa. Arch Dermatol 1982;118:375-84.

5 Bar-Maor JA, Nissan S, Nevo S. Pyloric atresia: a hereditary congenital anomaly with autosomal recessive transmission. $\mathcal{f}$ Med Genet 1972;9:70-2.

6 Weber M. Hemidesmosome deficiency of gastro-intestinal mucosa, demonstrated in a child with Herlitz syndrome and pyloric atresia. Acta Dermatol Venereol (Stockh) 1987;67:360-2.

7 Anton-Lamprecht I, Arnold ML. Prenatal diagnosis of inherited epidermolyses. Curr Probl Dermatol 1987;16:146-57.

8 Heagerty AH, Kennedy AR, Eady RA, et al. GB3 monoclonal antibody for diagnosis of junctional epidermolysis bullosa. Lancet 1986;i:860.

9 Heagerty AHM, Eady RAJ, Kennedy AR, et al. Rapid prenatal diagnosis of epidermolysis bullosa letalis using GB3 monoclonal antibodies. Br $\mathcal{F}$ Dermatol 1987;117:271-5. 